TRANSACTIONS OF THE

AMERICAN MATHEMATICAL SOCIETY

Volume 364, Number 10, October 2012, Pages 5469-5489

S 0002-9947(2012)05668-6

Article electronically published on May 29, 2012

\title{
VERTICAL VERSUS CONICAL SQUARE FUNCTIONS
}

\author{
PASCAL AUSCHER, STEVE HOFMANN, AND JOSÉ-MARÍA MARTELL
}

Abstract. We study the difference between vertical and conical square functions in the abstract and also in the specific case where the square functions come from an elliptic operator.

\section{INTRODUCTION}

The purpose of this article is to draw attention to differences between vertical and conical square functions. By vertical square functions, we mean the usual Littlewood-Paley-Stein functionals. By conical square functions, we mean the area functionals of Lusin type. Our interest in this subject was triggered by the recent work of Dragičević and Volberg [DV]. Let us first describe what they proved.

Let $A=A(x)$ be an $n \times n$ matrix of complex $L^{\infty}$ coefficients, defined on $\mathbb{R}^{n}$, and satisfying

$$
\lambda|\xi|^{2} \leq \operatorname{Re} A \xi \cdot \bar{\xi} \text { and }|A \xi \cdot \bar{\zeta}| \leq \Lambda|\xi||\zeta|,
$$

for $\xi, \zeta \in \mathbb{C}^{n}$ and for some $\lambda, \Lambda$ such that $0<\lambda \leq \Lambda<\infty$. We define a second order divergence form operator

$$
L f \equiv-\operatorname{div}(A \nabla f),
$$

which we interpret in the sense of maximal accretive operators via a sesquilinear form.

Proposition 1.1 (DV]). If $A$ is real and $1<p<\infty$, there is a dimension free bilinear estimate

$$
\iint_{\mathbb{R}_{+}^{n+1}}\left|\nabla_{y} e^{-t L} f(y)\left\|\nabla_{y} e^{-t L} g(y) \mid d y d t \leq C(p, \lambda, \Lambda)\right\| f\left\|_{p}\right\| g \|_{p^{\prime}}\right.
$$

Here $p^{\prime}$ is the conjugate exponent to $p$.

Setting aside the dimension free bound, this result is striking in view of the following vertical square function estimate.

Received by the editors December 18, 2010.

2010 Mathematics Subject Classification. Primary 42B25.

Key words and phrases. Vertical square functions, conical square functions, extrapolation, elliptic operators.

Part of this work was carried out while the first author was visiting the Centre for Mathematics and its Applications, Australian National University, Canberra ACT 0200, Australia.

The second author was partially supported by NSF grant number DMS 0801079.

The third author was supported by MEC Grant MTM2010-16518. 


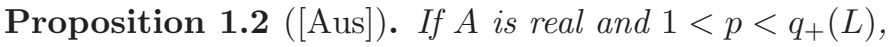

$$
\int_{\mathbb{R}^{n}}\left(\int_{0}^{\infty}\left|\nabla_{y} e^{-t L} f(y)\right|^{2} d t\right)^{p / 2} d y \leq C(p, n, \lambda, \Lambda)^{p}\|f\|_{p}^{p}
$$

Furthermore, this estimate fails for $p>q_{+}(L)$ (if $\left.q_{+}(L)<\infty\right)$.

The number $q_{+}(L)$ has been introduced in $\mathrm{Aus}$, as well as the three other numbers $p_{-}(L), q_{-}(L)$ and $p_{+}(L)$ as limits of the following intervals. The interval $\left(p_{-}(L), p_{+}(L)\right)$ is the maximal open interval where the heat semigroup $\left\{e^{-t L}\right\}_{t>0}$ is uniformly bounded on $L^{p}$ or equivalently the semigroup satisfies $L^{p}-L^{q}$ offdiagonal estimates when $p_{-}(L)<p \leq q<p_{+}(L)$; see (3.1) below. Analogously, $\left(q_{-}(L), q_{+}(L)\right)$ is the maximal open interval where $\left\{\sqrt{t} \nabla e^{-t L}\right\}_{t>0}$ is uniformly bounded on $L^{p}$ or satisfies $L^{p}-L^{q}$ off-diagonal estimates when $q_{-}(L)<p \leq q<$ $q_{+}(L)$. These intervals also determine up to endpoints the range of $L^{p}$ boundedness of the functional calculus, Riesz transform and vertical square functions. One has $p_{-}(L)=q_{-}(L),\left(q_{+}(L)\right)^{*} \leq p_{+}(L)$, where $q^{*}=q n /(n-q)$ when $q<n$ and $q^{*}=\infty$ otherwise. Also, $p_{-}(L)=q_{-}(L)=1$ and $p_{+}(L)=q_{+}(L)=\infty$ if $n=1$. For $n=2$, or for $n \geq 3$ and $L$ with real coefficients, the same is true except that one can only say that $q_{+}(L)>2$ for $n \geq 2$ and this is sharp. Additionally, if $n \geq 3$ and $A$ has complex coefficients, then $p_{-}(L)<2 n /(n+2)$ and $p_{+}(L)>2 n /(n-2)$. See Aus for full details. Also HMMc proves the latter inequalities to be sharp using an example of Freshe Fre.

Since $q_{+}(L)$ can be arbitrarily close to 2 , one cannot deduce the bilinear estimate in Proposition 1.1 from the vertical square function estimate. So the bilinear estimate seems to exhibit some special features that the vertical square function does not have. Indeed, bilinear integrals as above can also be estimated using conical square functions thanks to an averaging trick that appears in [FS] and [CMS]:

$$
\iint_{\mathbb{R}_{+}^{n+1}} F(y, t) G(y, t) d y d t=b_{n}^{-1} \int_{\mathbb{R}^{n}}\left(\iint_{\mathbb{R}_{+}^{n+1}} F(y, t) G(y, t) h\left(\frac{x-y}{t^{1 / 2}}\right) \frac{d y d t}{t^{n / 2}}\right) d x
$$

with $h$ the indicator function of the unit ball and $b_{n}$ its volume, so that

$$
\left|\iint_{\mathbb{R}_{+}^{n+1}} F(y, t) G(y, t) d y d t\right| \leq c_{n}\left\|S_{h} F\right\|_{p}\left\|S_{h} G\right\|_{p^{\prime}}
$$

with

$$
S_{h} F(x)=\left(\iint_{|x-y|<\sqrt{t}}|F(y, t)|^{2} \frac{d y d t}{t^{n / 2}}\right)^{1 / 2} .
$$

Hence, applying this to $F(y, t)=\nabla_{y} e^{-t L} f(y)$ and $G(y, t)=\nabla_{y} e^{-t L} g(y)$ it becomes natural to expect that the corresponding conical square function estimate holds in a larger range of $p$ than the one for (1.2). Indeed, we shall show the following as part of Theorem 3.1

Proposition 1.3. If $A$ is real and $1<p<\infty$,

$$
\int_{\mathbb{R}^{n}}\left(\iint_{|x-y|<\sqrt{t}}\left|\nabla_{y} e^{-t L} f(y)\right|^{2} \frac{d y d t}{t^{n / 2}}\right)^{p / 2} d x \leq C(p, n, \lambda, \Lambda)^{p}\|f\|_{p}^{p} .
$$


Thus (1.1) holds at least with a dimension dependent bound. We shall also study (1.3) for all complex $A$ and show that it holds when $p_{-}(L)<p<\infty$ and fails when $p<p_{-}(L)$ (if $p_{-}(L)>1$ ). This is consistent as $p_{-}(L)=1$ when $A$ is real. This also improves Aus, Corollary 6.10], where (1.3) was obtained in the range $p_{-}(L)<p<q_{+}(L)$. The bilinear inequality as in Proposition 1.1 then holds for a restricted range $p_{-}(L)<p<p_{-}(L)^{\prime}=p_{+}\left(L^{*}\right)$.

This leads us to the main point of this article about comparison between vertical and conical square functions. Propositions 1.1 and 1.3 show that the ranges of $p$ below 2 are the same but differ above 2. One may wonder whether there is an abstract principle behind this. But this is not the case. Aside from $p=2$ for which the averaging trick yields that they are equivalent, vertical and conical square functions only compare one way for $p \neq 2$ in the sense that one is automatically controlled by the other and simple examples show that the converses fail. More precisely, for $p>2$, it is well known and we shall recall why in Section 2, that a vertical square function controls the corresponding conical one. We shall also prove, and it seems this is not in the literature, that for $p<2$, the conical square function controls the corresponding vertical one. Comparing the ranges for (1.3) and (1.2) already furnishes a counterexample for the converse in the $p>2$ range and an example where the converse holds in the $p<2$ range. We note this can be done on a space of homogeneous type. We shall also study some weighted comparisons using extrapolation.

We finish this introduction by the following observations. As explained before, the range of $p$ for (1.2) is tight to the range of $L^{p}$ boundedness for $\sqrt{t} \nabla e^{-t L}$. As the $p<2$ range for (1.3) is a priori smaller than or equal to the $p<2$ range for (1.2), we obtain the best possible result by showing they are equal. For $p>2$ we exhibit a new phenomenon.

Our results show that the $p>2$ range for (1.3) is linked to the rate of decay in the $L^{2}$ off-diagonal estimates. If the latter is fast enough, then one obtains the full range $(2, \infty)$ as is the case in (1.3). In fact, this $L^{p}$ estimate amounts to proving boundedness of some vector-valued operator from $L^{p}$ into the parabolic version of the tent space $T_{2}^{p}$ of Coifman, Meyer, and Stein CMS. When the $L^{2}$ off-diagonal decay is fast enough we can prove, basically following the Fefferman-Stein argument, that $\left|\nabla_{y} e^{-t L} f(y)\right|^{2} d y d t$ is a parabolic Carleson measure for $f \in L^{\infty}$, which is nothing but an $L^{\infty} \rightarrow T_{2}^{\infty}$ estimate. One can then interpolate for $2<p<\infty$. When the rate of decay is slow (for example polynomial with small exponent) this argument does not seem to adapt and one needs other tools. This is the case for the conical square function based on $\varphi\left(t^{2} L\right)$ when $A$ is complex and $\varphi$ is not smooth at the origin. An example is the Poisson semigroup since $\varphi(z)=e^{-z^{1 / 2}}$ in this case. A different ingredient then comes into play, which is the decay at 0 of $\varphi$ or the order at which it vanishes, combined with the definition of $p_{+}(L)$. For instance, in Section 3.4 below, we shall prove the following and this is the $p>2$ range that is interesting for our discussion here.

Proposition 1.4. For $m$ a non-negative integer and $f \in L^{p}$, then

$$
\int_{\mathbb{R}^{n}}\left(\iint_{|x-y|<t}\left|t \nabla_{y, t}\left(\left(t^{2} L\right)^{m} e^{-t L^{1 / 2}} f\right)(y)\right|^{2} \frac{d y d t}{t^{n+1}}\right)^{p / 2} d x \leq C\|f\|_{p}^{p}
$$


whenever

$$
p_{-}(L)<p<\frac{n p_{+}(L)}{n-(2 m+1) p_{+}(L)} .
$$

For $(2 m+1) p_{+}(L) \geq n$, then the right hand side in (1.5) becomes $\infty$.

If $L$ had been the Laplacian, $2 m+1$ would just be the number of vanishing moments for the kernel of the convolution operator $\nabla_{y, t}\left(t^{2} L\right)^{m} e^{-t L^{1 / 2}}$ or the number of derivatives in front of the semigroup. Here we have $2 m$ th order "vanishing" coming from the exponent of the second order operator $L$, and +1 comes from the gradient.

A few comments are in order. We first point out that, since vertical and conical square functions are equivalent in $L^{2}$, one may integrate by parts in $t$ and use properties of the semigroup to pass from any choice of non-negative integer $m$ to another, in the case $p=2$. In fact, one may even take $m$ of the form $m=k+1 / 2$, with $k$ a non-negative integer. For $p>2$, different values of $m$ apparently need no longer be equivalent; the conclusion of the proposition yields a better range of $p$ for larger $m$ (up to the critical value with $(2 m+1) p_{+}(L)=n$ ).

In particular, the case $m=0$ of (1.4) gives standard area integral estimates for weak solutions of the equation

$$
\partial_{t}^{2} u+\operatorname{div} A \nabla u=0 .
$$

When $A$ is real (in which case $p_{-}(L)=1, p_{+}(L)=\infty$ ), such estimates may be obtained as a special case (the "block matrix" case) of the result of Dahlberg, Jerison, and Kenig in DJK], using the fact that one has non-tangential estimates for the solutions $u(\cdot, t):=e^{-t \sqrt{L}} f$ in every $L^{p}, 1<p \leq \infty$. The present argument allows for a direct (and simpler) proof than that in DJK] in this special case. Moreover, it has the added virtue of applying to the case of complex coefficients. Of course, we do not address the question of "full" coefficient matrices (i.e., those that need not be in block form), as is done in DJK.

\section{VeRTICAL VERSUS CONICAL}

For a locally square integrable function $f$ on $\mathbb{R}_{+}^{n+1}$, denote

$$
S f(x)=\left(\iint_{|x-y|<t}|f(y, t)|^{2} \frac{d y d t}{t^{n+1}}\right)^{1 / 2}, \quad x \in \mathbb{R}^{n},
$$

and

$$
V f(x)=\left(\int_{t>0}|f(x, t)|^{2} \frac{d t}{t}\right)^{1 / 2}, \quad x \in \mathbb{R}^{n} .
$$

$S f$ is lower semi-continuous hence a measurable function. Measurability on $V f$ follows from the local square integrability of $f$.

We remark that

$$
\|S f\|_{2}^{2}=b_{n}\|V f\|_{2}^{2}
$$

with $b_{n}$ the volume of the unit Euclidean ball. 


\subsection{Comparison in Lebesgue spaces.}

Proposition 2.1. Let $f$ be locally square integrable on $\mathbb{R}_{+}^{n+1}$.

(a) For $2<p<\infty$,

$$
\|S f\|_{p} \leq C(p, n)\|V f\|_{p}
$$

(b) For $0<p<2$,

$$
\|V f\|_{p} \leq C(p, n)\|S f\|_{p}
$$

(c) The converses fail for all $p \neq 2$.

Proof. Part $(a)$ is standard and appears already in [St, p. 91]. For the sake of self-containment, we recall the argument. As $p>2, q=p / 2>1$, and we can estimate $\|S f\|_{p}=\left\|(S f)^{2}\right\|_{p / 2}^{2}$ by dualizing against a function $h \in L^{q^{\prime}}$. Now, the averaging trick applies and yields

$$
\begin{aligned}
\int_{\mathbb{R}^{n}}(S f)^{2}(x) h(x) d x & =b_{n} \iint_{\mathbb{R}_{+}^{n+1}}|f(y, t)|^{2}\left(\frac{1}{|B(y, t)|} \int_{B(y, t)} h(x) d x\right) \frac{d y d t}{t} \\
& \leq b_{n} \int_{\mathbb{R}^{n}}(V f)^{2}(y) M h(y) d y \\
& \leq b_{n}\|V f\|_{p}^{2}\|M h\|_{q^{\prime}}
\end{aligned}
$$

and one concludes using the boundedness of the maximal operator over balls $M$ in $L^{q^{\prime}}$.

Let us now prove Part (b). Fix $0<p<2$, $f$ with $S f \in L^{p}$ and $\lambda>0$. Since $x \mapsto S f(x)$ is lower semi-continuous, the set $O=\{S f>\lambda\}$ is open. Let $F$ be the complement of $O$ in $\mathbb{R}^{n}, R(F)$ be the union of the cones $|x-y|<t$ with vertices $x \in F$. We also set $\tilde{O}=\left\{x \in \mathbb{R}: M\left(\chi_{O}\right)(x)>1 / 2\right\}$ and $\tilde{F}=\mathbb{R}^{n} \backslash \tilde{O}$. We note that $O \subset \tilde{O}$ since $O$ is open, and thus $\tilde{F} \subset F$. If $y \in \tilde{F}$ and $t>0$ we have $|O \cap B(y, t)| /|B(y, t)| \leq 1 / 2$ and consequently, $|F \cap B(y, t)| / t^{n} \geq b_{n} / 2$. Hence,

$$
\begin{aligned}
\int_{F}(S f)^{2}(x) d x & =\int_{x \in F} \iint_{|x-y|<t}|f(y, t)|^{2} \frac{d x d y d t}{t^{n+1}} \\
& =\iint_{R(F)} \frac{|F \cap B(y, t)|}{t^{n}}|f(y, t)|^{2} \frac{d y d t}{t} \\
& \geq \frac{b_{n}}{2} \int_{y \in \tilde{F}} \int_{t>0}|f(y, t)|^{2} \frac{d y d t}{t} \\
& =\frac{b_{n}}{2} \int_{\tilde{F}}(V f)^{2}(y) d y \\
& \geq \frac{b_{n}}{2} \lambda^{2}|\{V f>\lambda\} \cap \tilde{F}|
\end{aligned}
$$

Besides, for $0<r<p$, using the weak type $(1,1)$ for $M$,

$$
|\{V f>\lambda\} \cap \tilde{O}| \leq|\tilde{O}| \leq 2 \cdot 3^{n}|O| \leq \frac{2 \cdot 3^{n}}{\lambda^{r}} \int_{O}(S f)^{r}(x) d x .
$$


Hence,

$$
\begin{aligned}
\int_{\mathbb{R}^{n}}(V f)^{p}(x) d x & =p \int_{0}^{\infty} \lambda^{p-1}|\{V f>\lambda\}| d \lambda \\
\leq & \frac{2 p}{b_{n}} \int_{0}^{\infty} \lambda^{p-2-1} \int_{S f \leq \lambda}(S f)^{2}(x) d x d \lambda \\
& \quad+2 p \cdot 3^{n} \int_{0}^{\infty} \lambda^{p-r-1} \int_{S f>\lambda}(S f)^{r}(x) d x d \lambda \\
& =\left(\frac{2 p}{b_{n}(2-p)}+\frac{2 p \cdot 3^{n}}{p-r}\right) \int_{\mathbb{R}^{n}}(S f)^{p}(x) d x .
\end{aligned}
$$

We now finish the proof with Part $(c)$. It is convenient to introduce

$$
\tilde{S} f(x)=\iint_{|x-y|<t}|f(y, t)| \frac{d y d t}{t^{n+1}}, \quad \tilde{V} f(x)=\int_{t>0}|f(x, t)| \frac{d t}{t} .
$$

Note that $S f=\tilde{S}\left(|f|^{2}\right)^{1 / 2}$ and $V f=\tilde{V}\left(|f|^{2}\right)^{1 / 2}$, so that, for a locally integrable function $f$ on $\mathbb{R}_{+}^{n+1}$, we seek to disprove the inequalities

$$
\begin{gathered}
\|\tilde{S} f\|_{p} \leq C\|\tilde{V} f\|_{p}, \quad 0<p<1 \\
\|\tilde{V} f\|_{p} \leq C\|\tilde{S} f\|_{p}, \quad 1<p<\infty
\end{gathered}
$$

We write the argument so that it is easy to adapt it to a space of homogeneous type, denoting by $v(B)$ the volume of a ball and using implicitly the doubling property in the argument. See Remark 2.2 below.

For (2.1) we consider $f_{N}(x, t)=N^{-1} t \chi(x) \chi_{0}(t / N)$ with $N \gg 1$ and where $\chi$ is the characteristic function of the unit ball $B(0,1)$ and $\chi_{0}$ denotes the characteristic function of the interval $[0,1]$. On the one hand,

$$
\tilde{V} f_{N}(x)=\int_{0}^{\infty}\left|f_{N}(x, t)\right| \frac{d t}{t}=N^{-1} \chi(x) \int_{0}^{N} d t=\chi(x),
$$

and therefore

$$
\left\|\tilde{V} f_{N}\right\|_{p}^{p}=v(B(0,1)) .
$$

On the other hand, fixed $|x| \leq N / 8$, if $|y| \leq 1$ we have $|x-y|<N / 4$ (provided $N>8$ ) and then

$$
\begin{aligned}
\tilde{S} f_{N}(x) & =N^{-1} \iint_{|x-y|<t} t \chi(y) \chi_{0}(t / N) \frac{d y d t}{t v(B(y, t))} \\
& =N^{-1} \int_{|y| \leq 1} \int_{|x-y|<t \leq N} \frac{d t d y}{v(B(x, t))} \\
& \geq N^{-1} \int_{|y| \leq 1} \int_{N / 4<t \leq N} \frac{d t d y}{v(B(x, t))} \\
& \geq C \frac{v(B(0,1))}{v(B(0, N))} .
\end{aligned}
$$

This implies that

$$
\left\|\tilde{S} f_{N}\right\|_{p}^{p} \geq \int_{|x| \leq N / 8} \tilde{S} f(x)^{p} d x \geq C \frac{v(B(0,1))^{p}}{v(B(0, N))^{p-1}} .
$$


Gathering the obtained estimates,

$$
\frac{\left\|\tilde{S} f_{N}\right\|_{p}^{p}}{\left\|\tilde{V} f_{N}\right\|_{p}^{p}} \geq C \frac{v(B(0, N))^{1-p}}{v(B(0,1))^{1-p}} .
$$

Thus 2.1) cannot hold as $v(B(0, N))$ increases to $\infty$ and $1-p>0$.

For (2.2) we consider $f_{N}(x, t)=t v(B(x, t)) \chi_{N}(x) \chi_{0}(t)$ with $N \gg 1$ where $\chi_{N}$ is the characteristic function of the ball of radius $1 / N$. We first calculate $\tilde{V} f_{N}$ :

$$
\begin{aligned}
\tilde{V} f_{N}(x) & =\int_{0}^{\infty}\left|f_{N}(x, t)\right| \frac{d t}{t} \\
& \geq \chi_{N}(x) \int_{1 / 2}^{1} v(B(x, t)) d t \\
& \geq C \chi_{N}(x) v(B(x, 1)) \\
& \geq C \chi_{N}(x) v(B(0,1))
\end{aligned}
$$

and therefore

$$
\left\|\tilde{V} f_{N}\right\|_{p}^{p} \geq C v(B(0,1 / N)) v(B(0,1))^{p} .
$$

We find an upper bound for $\left\|\tilde{S} f_{N}\right\|_{p}$. We notice that if $|x|>2,|y| \leq 1 / N$ and $0 \leq t \leq 1$ we have $|x-y|>1 \geq t$ (if $N \geq 1$ ). Thus, $\tilde{S} f_{N}(x)=0$ if $|x|>2$. On the other hand, for all $x \in \mathbb{R}^{n}$ :

$$
\tilde{S} f_{N}(x)=\iint_{|x-y|<t \leq 1} \chi_{N}(y) d y d t \leq v(B(0,1 / N)) .
$$

Then, we obtain

$$
\left\|\tilde{S} f_{N}\right\|_{p}^{p}=\int_{|x| \leq 2} \tilde{S} f_{N}(x)^{p} d x \leq C v(B(0,1 / N))^{p} v(B(0,1))
$$

so that

$$
\frac{\left\|\tilde{S} f_{N}\right\|_{p}^{p}}{\left\|\tilde{V} f_{N}\right\|_{p}^{p}} \leq C \frac{v(B(0,1 / N))^{p-1}}{v(B(0,1))^{p-1}},
$$

which goes to 0 as $N \rightarrow \infty$ if $p>1$.

Remark 2.2. The reader can notice that this theorem generalizes to spaces of homogeneous type $X$ with infinite volume and at least one point that is not an atom (which plays the role of 0 ). That is, $\mathbb{R}_{+}^{n+1}$ is changed to $X \times \mathbb{R}_{+}$and in the definition of $S f(x)$ the measure has to change to $\frac{d \mu(y) d t}{t \mu(B(y, t))}$.

2.2. Weighted estimates via extrapolation. Let us present a weighted version of Proposition 2.1 using extrapolation. That is, $L^{2}$ estimates with suitable Muckenhoupt weights imply $L^{p}$ comparisons in weighted spaces. Let $A_{p}, 1 \leq p<\infty$, denote the classical Muckenhoupt classes of weights and $R H_{p}, 1<p \leq \infty$, the class of reverse Hölder weights. See for example [AM]. Again everything extends to a space of homogeneous type as in the remark above. We stick to the Euclidean space for simplicity.

Proposition 2.3. Let $f$ be a locally square integrable function on $\mathbb{R}_{+}^{n+1}$.

(a) For $2<p<\infty$ and $w \in A_{p / 2}$,

$$
\|S f\|_{L^{p}(w)} \leq C(p, w)\|V f\|_{L^{p}(w)} .
$$


(b) For $0<p<2$ and $w \in R H_{(2 / p)^{\prime}}$,

$$
\|V f\|_{L^{p}(w)} \leq C(p, w)\|S f\|_{L^{p}(w)} .
$$

Proof. We begin with Part $(a)$. Given any $w \in A_{\infty}$ we easily have

$$
\begin{aligned}
\|S f\|_{L^{2}(w)}^{2} & =\int_{\mathbb{R}^{n}} \iint_{|x-y|<t}|f(y, t)|^{2} \frac{d y d t}{t^{n+1}} w(x) d x \\
& =b_{n} \int_{\mathbb{R}^{n}} \int_{0}^{\infty}|f(y, t)|^{2} \frac{w(B(y, t))}{|B(y, t)|} \frac{d y d t}{t} .
\end{aligned}
$$

We note that if $w \in A_{1}$, that is, $M w(y) \leq[w]_{A_{1}} w(y)$ for a.e. $y \in \mathbb{R}^{n}$, then we have for all $t>0$,

$$
\frac{w(B(y, t))}{|B(y, t)|} \leq M w(y) \leq[w]_{A_{1}} w(y), \quad \text { a.e. } y \in \mathbb{R}^{n} .
$$

Then, we obtain

$$
\|S f\|_{L^{2}(w)}^{2} \leq b_{n}[w]_{A_{1}} \int_{\mathbb{R}^{n}} \int_{0}^{\infty}|f(y, t)|^{2} \frac{d t}{t} w(y) d y=b_{n}[w]_{A_{1}}\|V f\|_{L^{2}(w)}^{2} .
$$

Next we invoke the Rubio de Francia extrapolation theorem (see [Rub], Gar] for the original result, and CMP1, CMP2 for a statement written in terms of pairs of functions) for the pairs $\left((S f)^{2},(V f)^{2}\right)$ : the starting estimate in $L^{1}(w)$ for every $w \in A_{1}$ implies that for every $2<p<\infty$ and $w \in A_{p / 2}$,

$$
\|S f\|_{L^{p}(w)} \leq C(p, w)\|V f\|_{L^{p}(w)} .
$$

Strictly speaking, the argument applies whenever the left hand side is finite. This is the case if $f$ is a priori bounded with compact support in $\mathbb{R}_{+}^{n+1}$. Monotone convergence implies that the inequality is valid for all locally square integrable functions $f$.

For the reverse estimate in Part $(b)$, we recall that $w \in R H_{\infty}$ if for every ball $B$ we have

$$
w(x) \leq[w]_{R H_{\infty}} \frac{1}{|B|} \int_{B} w(y) d y, \quad \text { a.e. } x \in B .
$$

Then, using Lebesgue's differentiation theorem we obtain that for a.e. $y \in \mathbb{R}^{n}$ and for all $t>0$,

$$
\begin{aligned}
w(y) & \leq \sup _{0<\tau \leq t} \frac{1}{|B(y, \tau)|} \int_{B(y, \tau)} w(x) d x \\
& \leq[w]_{R H_{\infty}} \sup _{0<\tau \leq t} \frac{1}{|B(y, \tau)|} \int_{B(y, \tau)} \frac{1}{|B(y, t)|} \int_{B(y, t)} w(z) d z d x \\
& =[w]_{R H_{\infty}} \frac{w(B(y, t))}{|B(y, t)|} .
\end{aligned}
$$

Thus, for every $w \in R H_{\infty}$, by (2.3) we have

$$
\begin{aligned}
\|V f\|_{L^{2}(w)}^{2} & =\int_{\mathbb{R}^{n}} \int_{0}^{\infty}|f(y, t)|^{2} \frac{d t}{t} w(y) d y \\
& \leq[w]_{R H_{\infty}} \int_{\mathbb{R}^{n}} \int_{0}^{\infty}|f(y, t)|^{2} \frac{w(B(y, t))}{|B(y, t)|} \frac{d y d t}{t} \\
& =[w]_{R H_{\infty}} b_{n}^{-1}\|S f\|_{L^{2}(w)}^{2} .
\end{aligned}
$$


Considering the pairs $(F, G)=\left((V f)^{2},(S f)^{2}\right)$ we have obtained that

$$
\int_{\mathbb{R}^{n}} F(x) w(x) d x \leq[w]_{R H_{\infty}} b_{n}^{-1} \int_{\mathbb{R}^{n}} G(x) w(x) d x, \quad \forall w \in R H_{\infty} .
$$

We take an arbitrary $p_{0}$ with $0<p_{0}<1$ and set $q_{0}=r=1$. Then the last estimate holds in particular for every $w \in A_{r / p_{0}} \cap R H_{\left(q_{0} / r\right)^{\prime}}$. We apply the extrapolation theorem for limited ranges [AM, Theorem 4.9] (see also [CMP2]) to conclude that for all $p_{0}<q<q_{0}$,

$$
\int_{\mathbb{R}^{n}} F(x)^{q} w(x) d x \leq C_{w} \int_{\mathbb{R}^{n}} G(x)^{q} w(x) d x, \quad \forall w \in A_{q / p_{0}} \cap R H_{\left(q_{0} / q\right)^{\prime}},
$$

whenever the left hand side is finite. This is the case when $f$ is bounded with compact support in $\mathbb{R}_{+}^{n+1}$ and can be removed by monotone convergence to allow all locally square integrable functions $f$. Next, we fix $0<q<1$ and $w \in R H_{(1 / q)^{\prime}}$. Then, $w \in A_{\infty}$ and there exists $0<p_{0}<q$ such that $w \in A_{q / p_{0}}$. Thus we can apply the last estimate since $0<p_{0}<q<1=q_{0}$ and $w \in A_{q / p_{0}} \cap R H_{\left(q_{0} / q\right)^{\prime}}$. Hence we have proved that for every $0<p<2$ and $w \in R H_{(2 / p)^{\prime}}$,

$$
\|V f\|_{L^{p}(w)} \leq C(p, w)\|S f\|_{L^{p}(w)} .
$$

Notice that from the argument one sees that the extrapolations take initial estimates in $L^{1}(w)$. Indeed, from the beginning one could have worked with the operators $\tilde{S}$ and $\tilde{V}$ defined above. The argument just presented shows that for every locally integrable function $f$ on $\mathbb{R}_{+}^{n+1}$, if $1 \leq p<\infty$ and $w \in A_{p}$, then

$$
\|\tilde{S} f\|_{L^{p}(w)} \leq C(p, w)\|\tilde{V} f\|_{L^{p}(w)},
$$

and if $0<p \leq 1$ and $w \in R H_{(1 / p)^{\prime}}$, then

$$
\|\tilde{V} f\|_{L^{p}(w)} \leq C(p, w)\|\tilde{S} f\|_{L^{p}(w)} .
$$

\section{SQUARE FUnCtions FOR TypicAl FUnCtions of $L$}

Consider the operator $L$ defined in the Introduction. We introduce the following conical and vertical square functions:

$$
\begin{gathered}
\mathcal{G}_{P}(f)(x)=\left(\iint_{|x-y|<t}\left|t \nabla_{y, t} e^{-t L^{1 / 2}} f(y)\right|^{2} \frac{d y d t}{t^{n+1}}\right)^{1 / 2}, \\
G_{P}(f)(y)=\left(\int_{t>0}\left|t \nabla_{y, t} e^{-t L^{1 / 2}} f(y)\right|^{2} \frac{d t}{t}\right)^{1 / 2}, \\
\mathcal{G}_{h}(f)(x)=\left(\iint_{|x-y|<\sqrt{t}}\left|\nabla_{y} e^{-t L} f(y)\right|^{2} \frac{d y d t}{t^{n / 2}}\right)^{1 / 2}, \\
G_{h}(f)(y)=\left(\int_{t>0}\left|\nabla_{y} e^{-t L} f(y)\right|^{2} d t\right)^{1 / 2} .
\end{gathered}
$$

The $P$ subscript refers to the fact that we are dealing with the Poisson semigroup $e^{-t L^{1 / 2}}$ for $L$. The $h$ subscript refers to the heat semigroup $e^{-t L}$. The curly letters are for the conical square functions and the capital letters are for the vertical ones. From our general observations we know that $\left\|\mathcal{G}_{P}(f)\right\|_{p} \lesssim\left\|G_{P}(f)\right\|_{p}$ for $2 \leq p<\infty$ and $\left\|G_{P}(f)\right\|_{p} \lesssim\left\|\mathcal{G}_{P}(f)\right\|_{p}$ for $0<p \leq 2$ and similarly for the heat versions by 
making a change of variables in $t$. Note that these square functions all contain a spatial gradient. Hence we are not working within the functional calculus of $L$.

We want to compare the $L^{p}$ norms of each square function with the $L^{p}$ norm of the original function $f$.

For $p=2$, a mere integration by parts (see Aus]) yields that

$$
\left\|G_{P}(f)\right\|_{2}+\left\|G_{h}(f)\right\|_{2} \approx C(\lambda, \Lambda)\|f\|_{2} .
$$

As seen above, conical square functions behave as the vertical ones in $L^{2}$.

We turn to a summary of results on $L^{p}$. Let $p^{*}=\frac{n p}{n-p}$ if $p<n$ and $\infty$ otherwise. Let us remind the reader that the exponents $p_{ \pm}(L), q_{ \pm}(L)$ were defined in the introduction, in the discussion following the statement of Proposition 1.2

Theorem 3.1. (1) $G_{h}$ is bounded on $L^{p}$ for $p_{-}(L)<p<q_{+}(L)$.

(2) $\mathcal{G}_{h}$ is bounded on $L^{p}$ for $p_{-}(L)<p<\infty$.

(3) $G_{P}$ is bounded on $L^{p}$ for $p_{-}(L)<p<q_{+}(L)$.

(4) $\mathcal{G}_{P}$ is bounded on $L^{p}$ for $p_{-}(L)<p<p_{+}(L)^{*}$.

The upper bounds are optimal except maybe for $\mathcal{G}_{P}$. The lower bounds are all optimal.

The converse estimates $\|f\|_{p} \lesssim\|g(f)\|_{p}$ are valid for all $p \in(1, \infty)$ and $f \in$ $L^{p} \cap L^{2}$ and all four square functions. Hence, each defines a new norm on $L^{p}$ for $p$ in the corresponding range above.

Fix $\mu \in(0, \pi / 2)$ and $1 \leq p \leq \infty$. We say, following Aus, that a family of linear operators $\left(T_{z}\right)_{z \in \Sigma_{\mu}}$ satisfies $L^{p}-L^{q}$ off-diagonal estimates if there exist constants $c, C$ such that for all $z \in \Sigma_{\mu}:=\left\{z \in \mathbb{C}^{*} ;|\arg z|<\mu\right\}$, all Borel sets $E, F$ and all $f \in L^{p}(E)$, we have

$$
\left\|T_{z}\left(f \chi_{E}\right)\right\|_{L^{q}(F)} \leq C|z|^{-\frac{n}{2}\left(\frac{1}{p}-\frac{1}{q}\right)} e^{-\frac{c d(E, F)^{2}}{|z|}}\|f\|_{L^{p}(E)} .
$$

This holds for $\mu<\pi / 2-\omega$ with $\omega$ the type of $L, T_{z}=(z L)^{m} e^{-z L}$ with $p_{-}(L)<$ $p \leq q<p_{+}(L)$ and $T_{z}=|z|^{1 / 2} \nabla(z L)^{m} e^{-z L}$ with $q_{-}(L)=p_{-}(L)<p \leq q<q_{+}(L)$, for any non-negative integer $m$. See [Aus, Chapter 3].

For $1 \leq p<\infty$, we recall that the tent space $T_{2}^{p}$ denotes the space of locally square integrable functions in $\mathbb{R}_{+}^{n+1}$ such that $S f \in L^{p}\left(\mathbb{R}^{n}\right)$ with the notation of Section 2, The norm in $T_{2}^{p}$ is given by $\|S f\|_{p}$ as defined in Section 2, Note that changing the aperture of cones yields equivalent norms. For $p=\infty$, we let $T_{2}^{\infty}$ be the space of locally square integrable functions in $\mathbb{R}_{+}^{n+1}$ such that

$$
\|f\|_{T_{2}^{\infty}}=\sup _{B}\left(\frac{1}{|B|} \iint_{B \times\left(0, r_{B}\right)}|f(y, t)|^{2} \frac{d y d t}{t}\right)^{1 / 2}<\infty,
$$

the supremum being taken above all balls, and $r_{B}$ denotes the radius of $B$. The spaces $T_{2}^{p}, 1 \leq p \leq \infty$, form a complex interpolation family. For more see CMS. Note that the $L^{p}$ boundedness of a conical square function reformulates canonically as an $L^{p}$ to $T_{2}^{p}$ boundedness.

We first prove boundedness and sharpness for each square function. We consider next the converse inequalities globally.

3.1. Proof of Theorem $\mathbf{3 . 1}$ for $G_{h}$. This was treated in Aus. There the range of $p$ is shown to be the largest possible open set. 
3.2. Proof of Theorem 3.1 for $\mathcal{G}_{h}$. For $p \leq 2$, it is in Aus. For $p=\infty$, we first obtain the boundedness of $f \mapsto t \nabla e^{-t^{2} L} f$ from $L^{\infty}$ to $T_{2}^{\infty}$ by a wellknown argument of Fefferman-Stein [FS. More precisely, we fix a ball $B$ and write $f=f_{\text {loc }}+f_{\text {glob }}$ where $f_{\text {loc }}=f \chi_{4 B}$. Using the $L^{2}$ boundedness of $\mathcal{G}_{h}$,

$$
\frac{1}{|B|} \iint_{\hat{B}}\left|t \nabla e^{-t^{2} L} f_{\mathrm{loc}}(x)\right|^{2} \frac{d x d t}{t} \lesssim \frac{1}{|B|}\left\|G_{h} f_{\mathrm{loc}}\right\|_{2}^{2} \lesssim \frac{1}{|B|}\left\|f_{\mathrm{loc}}\right\|_{2}^{2} \lesssim\|f\|_{\infty}^{2} .
$$

Next, the off-diagonal decay (3.1) with $p=q=2$ for $t^{1 / 2} \nabla e^{-t L}$ implies for some $0<c, C<\infty$,

$$
\frac{1}{|B|} \int_{B}\left|t \nabla e^{-t^{2} L} f_{\text {glob }}(x)\right|^{2} d x \leq C \sum_{j \geq 2} e^{-\frac{c 4^{j} r_{B}^{2}}{t^{2}}} f_{2^{j+1} B}|f(x)|^{2} d x,
$$

which, integrated against $d t / t$ in $t \in\left(0, r_{B}\right)$, yields a bound by $\|f\|_{\infty}^{2}$.

Then interpolate this estimate with the boundedness from $L^{2}$ to $T_{2}^{2}$ to get the boundedness from $L^{p}$ to $T_{2}^{p}$, which is the same as the $L^{p}$ boundedness of $\mathcal{G}_{h}$ by rescaling $t^{2} \mapsto t$ in the integrals.

Note that compared to $\mathrm{AuS}$, the upper bound improves from $q_{+}(L)$ to $\infty$ and is of course optimal. As for the lower bounds, we have $\left\|G_{h} f\right\|_{p} \lesssim\left\|\mathcal{G}_{h} f\right\|_{p}$ when $p \leq 2$. Hence the fact that $p_{-}(L)$ is optimal for $G_{h}$ (see Aus]) implies the same for $\mathcal{G}_{h}$.

3.3. Proof of Theorem 3.1 for $G_{P}$. We begin with removing the $\nabla$ part in $G_{P}$ when $q_{-}(L)<p<q_{+}(L)$. We know that $\nabla L^{-1 / 2}$ is bounded on $L^{p}$ for $p$ in this range Aus. So by vector-valued (in the Hilbert space $H=L^{2}\left(\mathbb{R}^{+}, d t / t\right)$ ) extension (Gra, Proposition 4.5.9]), we have that

$$
\left\|G_{P} f\right\|_{p} \leq C\left\|g_{P} f\right\|_{p}, \quad q_{-}(L)<p<q_{+}(L)
$$

with

$$
g_{P}(f)(x)=\left(\int_{0}^{\infty}\left|t L^{1 / 2} e^{-t L^{1 / 2}} f(x)\right|^{2} \frac{d t}{t}\right)^{1 / 2} .
$$

Next, [HM Lemma 7.2], using the subordination formula

$$
e^{-t L^{1 / 2}} f=C \int_{0}^{\infty} \frac{e^{-s}}{\sqrt{s}} e^{-\frac{t^{2} L}{4 s}} f d s
$$

proves the pointwise inequality $g_{P} \leq C \tilde{g}_{h}$ with

$$
\tilde{g}_{h}(f)(x)=\left(\int_{0}^{\infty}\left|t^{2} L e^{-t^{2} L} f(x)\right|^{2} \frac{d t}{t}\right)^{1 / 2},
$$

and the latter is bounded on $L^{p}$ for $p_{-}(L)<p<p_{+}(L)$. This can be proved by adapting line by line [Aus, Theorem 6.1]. This also follows from Le Merdy's theorem [LeM, Corollary 2.3].

We conclude by noticing that $p_{-}(L)=q_{-}(L), q_{+}(L)<p_{+}(L)$ (when $q_{+}(L)<$ $\infty)$. This finishes the proof.

That the bounds $p_{-}(L)$ and $q_{+}(L)$ are sharp follows by the same argument as for Step 7 in Aus, Theorem 6.1]. 
3.4. Proof of Theorem 3.1 for $\mathcal{G}_{P}$. More generally, we shall discuss here the proof of Proposition 1.4 for which Theorem 3.1 (4) represents the case $m=0$. We shall treat the case $m>0$ explicitly only when $p>2$, as the cases $m=0$ and $m>0$ may be treated by the same argument when $p \leq 2$.

This part of the proof of Theorem 3.1 (and more generally, the proof of Proposition (1.4) is the most involved as it does not follow from other known arguments in a simple way. For $p=2$, this is classical integration by parts. We then present arguments for $p_{-}(L)<p<2$ and $2<p<p_{+}(L)^{*}$. That $p_{-}(L)$ is sharp follows by the same argument as for $\mathcal{G}_{h}$.

3.4.1. $p<2$. We present two proofs. The first one uses the recent theory of Hardy spaces adapted to $L$ from [HM, HMMc or from [BZ], and the second one adapts arguments in Aus to prove instead weak-type bounds. We remark as above that the same proofs apply to prove (1.4). We shall omit the details and stick to $m=0$.

Proof 1. Consider the Hardy spaces $H_{L}^{p}$ defined in [HM] for $p=1$ and [HMMc] for $p \geq 1$. The $H_{L}^{1} \rightarrow L^{1}$ boundedness of $\mathcal{G}_{P}$ is exactly as in [HM, Theorem 5.6]. Then interpolation (see [HMMc, Lemma 4.24]) with the $p=2$ case shows $H_{L}^{p} \rightarrow L^{p}$ boundedness of $\mathcal{G}_{P}$. Finally, identification of $H_{L}^{p}$ with $L^{p}$ if and only if $p_{-}(L)<p<p_{+}(L)$ is proved in [HMMc, Proposition 9.1] and concludes the argument.

We mention that one can also use the abstract Hardy spaces developed by Bernicot and Zhao in $\mathrm{BZ}$ and the interpolation further developed in Be1. Namely it suffices to prove an $L^{1}$ estimate on some abstract atoms (that is, an $H_{F, \text { ato }}^{1}$ to $L^{1}$ estimate with $H_{F, \text { ato }}^{1}$ as in Section 3.3 of [BZ]) and then interpolate. By checking details and values (left to the readers) from the clear presentation in $\mathrm{Be} 2$, one exactly finds the range for $L^{p}$ boundedness when $p<2$. This theory, compared to the [HM] theory, has the advantage of not caring much about the "right" definition of the Hardy spaces as this is not needed for the purpose of interpolation.

Proof 2. We proceed as in Aus, p. 61]. We need to adapt the proof of Aus, Theorem 1.1] to the present situation. We take $A_{r}=I-\left(I-e^{-r^{2} L}\right)^{N}$ with $N \geq 1$ an integer to be chosen and follow the proof of that result with $T=\mathcal{G}_{P}$ and $p_{-}(L)<p<2$. As $\mathcal{G}_{P}$ is bounded on $L^{2}$ and $A_{r}$ satisfies off-diagonal estimates in the range $\left(p_{-}(L), 2\right]$ it suffices to show that

$$
I=\left|\left\{x \in \mathbb{R}^{n} \backslash \bigcup_{i} 4 Q_{i}: \mathcal{G}_{P}\left(\sum_{i} h_{i}\right)(x)>\alpha / 3\right\}\right| \leq \frac{C}{\alpha^{p}} \int_{\mathbb{R}^{n}}|f(x)|^{p} d x,
$$

where $h_{i}=\left(I-A_{r_{i}}\right) b_{i}$ and $r_{i}$ is the sidelength of the cube $Q_{i}$ given by the CalderónZygmund lemma [Aus, Lemma 1.3]. We use Chebichev and Fubini:

$$
\begin{aligned}
I & \leq \frac{9}{\alpha^{2}} \int_{\mathbb{R}^{n} \backslash \bigcup_{i} 4 Q_{i}} \mathcal{G}_{P}\left(\sum_{i} h_{i}\right)(x)^{2} d x \\
& =\frac{9}{\alpha^{2}} \int_{\mathbb{R}^{n} \backslash \bigcup_{i} 4 Q_{i}} \iint_{|x-y|<t}\left(\sum_{i}\left|t \nabla_{y, t} e^{-t L^{1 / 2}} h_{i}(y)\right|\right)^{2} \frac{d y d t}{t^{n+1}} d x \\
& =\frac{9}{\alpha^{2}} \iint_{\mathbb{R}_{+}^{n+1}}\left(\sum_{i}\left|t \nabla_{y, t} e^{-t L^{1 / 2}} h_{i}(y)\right|\right)^{2} \frac{\left|B(y, t) \backslash \bigcup_{i} 4 Q_{i}\right|}{t^{n}} \frac{d y d t}{t}
\end{aligned}
$$




$$
\begin{aligned}
& \lesssim \frac{1}{\alpha^{2}} \iint_{\mathbb{R}_{+}^{n+1}}\left(\sum_{i} \chi_{2} Q_{i}(y)\left|t \nabla_{y, t} e^{-t L^{1 / 2}} h_{i}(y)\right|\right)^{2} \frac{\left|B(y, t) \backslash \bigcup_{i} 4 Q_{i}\right|}{t^{n}} \frac{d y d t}{t} \\
& +\frac{1}{\alpha^{2}} \iint_{\mathbb{R}_{+}^{n+1}}\left(\sum_{i} \chi_{\mathbb{R}^{n} \backslash 2 Q_{i}}(y)\left|t \nabla_{y, t} e^{-t L^{1 / 2}} h_{i}(y)\right|\right)^{2} \frac{\left|B(y, t) \backslash \bigcup_{i} 4 Q_{i}\right|}{t^{n}} \frac{d y d t}{t} \\
& =\frac{1}{\alpha^{2}}\left(I_{\text {loc }}+I_{\text {glob }}\right) .
\end{aligned}
$$

We estimate $I_{\text {loc }}$. Notice that since $y \in 2 Q_{i}$ we have that $B(y, t) \subset 4 Q_{i}$ for $t \leq c r_{i}$. Then, using that the collection $\left\{2 Q_{i}\right\}_{i}$ has bounded overlapping, we obtain

$$
\begin{aligned}
I_{\mathrm{loc}} & \lesssim \int_{\mathbb{R}^{n}} \int_{c r_{i}}^{\infty}\left(\sum_{i} \chi_{2} Q_{i}(y)\left|t \nabla_{y, t} e^{-t L^{1 / 2}} h_{i}(y)\right|\right)^{2} \frac{d y d t}{t} \\
& \lesssim \sum_{i} \int_{2 Q_{i}} \int_{c r_{i}}^{\infty}\left|t \nabla_{y, t} e^{-t L^{1 / 2}} h_{i}(y)\right|^{2} \frac{d y d t}{t} \\
& \lesssim \sum_{i}\left(\int_{c r_{i}}^{\infty} \int_{\mathbb{R}^{n}}\left|t \nabla_{y} e^{-t L^{1 / 2}} h_{i}(y)\right|^{2} \frac{d y d t}{t}\right. \\
& \left.+\int_{c r_{i}}^{\infty} \int_{\mathbb{R}^{n}}\left|t L^{1 / 2} e^{-t L^{1 / 2}} h_{i}(y)\right|^{2} \frac{d y d t}{t}\right) \\
\lesssim & \sum_{i} \int_{c r_{i}}^{\infty} \int_{\mathbb{R}^{n}}\left|t L^{1 / 2} e^{-t L^{1 / 2}} h_{i}(y)\right|^{2} \frac{d y d t}{t}
\end{aligned}
$$

where we have used the solution of the Kato conjecture AHLMcT to replace $\nabla_{y}$ by $L^{1 / 2}$. Next we use the subordination formula (3.2), Minkowski's inequality and the change of variable $t \mapsto t^{\prime}:=t^{2} / 4 s$,

$$
\begin{aligned}
\left(\int_{c r_{i}}\right. & \left.\int_{\mathbb{R}^{n}}\left|t L^{1 / 2} e^{-t L^{1 / 2}} h_{i}(y)\right|^{2} \frac{d y d t}{t}\right)^{1 / 2} \\
& \lesssim \int_{0}^{\infty} e^{-s}\left(\int_{c r_{i}}^{\infty} \int_{\mathbb{R}^{n}} \frac{t^{2}}{4 s}\left|L^{1 / 2} e^{-\frac{t^{2} L}{4 s}} h_{i}(y)\right|^{2} \frac{d y d t}{t}\right)^{1 / 2} d s \\
& \lesssim \int_{0}^{\infty} e^{-s}\left(\int_{c r_{i}^{2} / s}^{\infty} \int_{\mathbb{R}^{n}}\left|(t L)^{1 / 2} e^{-t L} h_{i}(y)\right|^{2} \frac{d y d t}{t}\right)^{1 / 2} d s
\end{aligned}
$$

Next we take $a=\frac{n}{p}-\frac{n}{2}$ and use the square function estimate of McIntosh-Yagi based on $(t L)^{(a+1) / 2} e^{-t L}$ :

$$
\begin{aligned}
\left(\int_{c r_{i}}\right. & \left.\int_{\mathbb{R}^{n}}\left|t L^{1 / 2} e^{-t L^{1 / 2}} h_{i}(y)\right|^{2} \frac{d y d t}{t}\right)^{1 / 2} \\
& \lesssim \int_{0}^{\infty} e^{-s}\left(\int_{c r_{i}^{2} / s}^{\infty} \int_{\mathbb{R}^{n}}\left|(t L)^{(a+1) / 2} e^{-t L} L^{-a} h_{i}(y)\right|^{2} t^{-a} \frac{d y d t}{t}\right)^{1 / 2} d s \\
& \lesssim \int_{0}^{\infty} e^{-s}\left(\frac{s}{r_{i}^{2}}\right)^{a / 2}\left(\int_{\mathbb{R}^{n}} \int_{0}^{\infty}\left|(t L)^{(a+1) / 2} e^{-t L}\left(L^{-a / 2} h_{i}\right)(y)\right|^{2} \frac{d t}{t} d y\right)^{1 / 2} d s \\
& \lesssim r_{i}^{-a}\left\|L^{-a / 2} h_{i}\right\|_{2} \lesssim r_{i}^{-a}\left\|h_{i}\right\|_{p}=r_{i}^{-a}\left\|\left(I-A_{r_{i}}\right) b_{i}\right\|_{p},
\end{aligned}
$$

where we have used [Aus, Proposition 5.3] in the last inequality. To conclude we use that $I-A_{r_{i}}=\left(I-e^{-r_{i}^{2} L}\right)^{N}$ is uniformly bounded on $L^{p}$ and the Calderón-Zygmund 
lemma

$$
\begin{aligned}
I_{\mathrm{loc}} & \lesssim \sum_{i} r_{i}^{-2 a}\left\|b_{i}\right\|_{p}^{2} \lesssim \alpha^{2} \sum_{i} r_{i}^{-2 a}\left|Q_{i}\right|^{2 / p} \lesssim \alpha^{2} \sum_{i}\left|Q_{i}\right| \\
& \lesssim \alpha^{2-p} \int_{\mathbb{R}^{n}}|f(x)|^{p} d x .
\end{aligned}
$$

Next we estimate $I_{\text {glob }}$. We write $C_{j}\left(Q_{i}\right)=2^{j+1} Q_{i} \backslash 2^{j} Q_{i}, j \geq 1$. By duality we can take a function $0 \leq H \in L^{2}\left(\mathbb{R}_{+}^{n+1}, \frac{d y d t}{t}\right)$ with norm 1 such that

$$
\begin{aligned}
I_{\text {glob }}^{1 / 2} & \lesssim\left(\iint_{\mathbb{R}_{+}^{n+1}}\left(\sum_{i} \chi_{\mathbb{R}^{n} \backslash 2 Q_{i}}(y)\left|t \nabla_{y, t} e^{-t L^{1 / 2}} h_{i}(y)\right|\right)^{2} \frac{d y d t}{t}\right)^{1 / 2} \\
& =\sum_{i} \int_{0}^{\infty} \int_{\mathbb{R}^{n} \backslash 2 Q_{i}}\left|t \nabla_{y, t} e^{-t L^{1 / 2}} h_{i}(y)\right| H(y, t) \frac{d y d t}{t} \\
& \lesssim \sum_{i} \sum_{j=1}^{\infty} 2^{j n}\left|Q_{i}\right| \int_{0}^{\infty} f_{C_{j}\left(Q_{i}\right)}\left|t \nabla_{y, t} e^{-t L^{1 / 2}} h_{i}(y)\right| H(y, t) \frac{d y d t}{t} \\
& \leq \sum_{i} \sum_{j=1}^{\infty} 2^{j n}\left|Q_{i}\right|\left(\int_{0}^{\infty} f_{C_{j}\left(Q_{i}\right)}\left|t \nabla_{y, t} e^{-t L^{1 / 2}} h_{i}(y)\right|^{2} \frac{d y d t}{t}\right)^{1 / 2} \\
& \times\left(\int_{0}^{\infty} f_{2^{j+1} Q_{i}} H(y, t)^{2} \frac{d y d t}{t}\right)^{1 / 2} \\
& \leq \sum_{i} \sum_{j=1}^{\infty} 2^{j n}\left|Q_{i}\right| I_{i j} \underset{y \in Q_{i}}{\operatorname{ess} \inf } M \tilde{H}(y)^{1 / 2},
\end{aligned}
$$

where $\tilde{H}(y)=\int_{0}^{\infty} H(y, t)^{2} d t / t$. We estimate $I_{i j}$ by the subordination formula, Minkowski's inequality and the change of variable $t \mapsto t^{\prime}:=t^{2} / 4 \mathrm{~s}$ :

$$
\begin{aligned}
I_{i j} \lesssim \int_{0}^{\infty} e^{-s}\left(\int_{0}^{\infty} f_{C_{j}\left(Q_{i}\right)}\left|\frac{t}{\sqrt{4 s}} \nabla_{y} e^{-\frac{t^{2} L}{4 s}} h_{i}(y)\right|^{2} \frac{d y d t}{t}\right)^{1 / 2} d s \\
\quad+\int_{0}^{\infty} e^{-s}\left(\int_{0}^{\infty} f_{C_{j}\left(Q_{i}\right)}\left|\frac{t}{\sqrt{4 s}} L^{1 / 2} e^{-\frac{t^{2} L}{4 s}} h_{i}(y)\right|^{2} \frac{d y d t}{t}\right)^{1 / 2} d s \\
\quad \lesssim \int_{0}^{\infty} e^{-s}\left(\int_{0}^{\infty} f_{C_{j}\left(Q_{i}\right)}\left|\sqrt{t} \nabla_{y} e^{-t L} h_{i}(y)\right|^{2} \frac{d y d t}{t}\right)^{1 / 2} d s \\
\quad+\int_{0}^{\infty} e^{-s}\left(\int_{0}^{\infty} f_{C_{j}\left(Q_{i}\right)}\left|(t L)^{1 / 2} e^{-t L} h_{i}(y)\right|^{2} \frac{d y d t}{t}\right)^{1 / 2} d s \\
\lesssim f_{C_{j}\left(Q_{i}\right)} G_{L}\left(\left(I-e^{-r_{i}^{2} L}\right)^{N} b_{i}\right)(y)^{2} d y+f_{C_{j}\left(Q_{i}\right)} g_{L}\left(\left(I-e^{-r_{i}^{2} L}\right)^{N} b_{i}\right)(y)^{2} d y \\
\lesssim 2^{-j n / 2} 4^{-N j} 2^{-j\left(\frac{n}{p}-\frac{n}{2}\right)}\left(f_{Q_{i}}\left|b_{i}(y)\right|^{p} d y\right)^{\frac{1}{p}} \\
\lesssim 2^{-j\left(2 N+\frac{n}{p}\right)} \alpha
\end{aligned}
$$

where in the next-to-last estimate we have used [Aus, pp. 55, 56] and the notation there for $G_{L}, g_{L}$ (the first one is here the same as $G_{h}$ ) and in the last one the Calderón-Zygmund lemma. Choosing $N$ such that $2 N+\frac{n}{p}-n>0$ we obtain by 
Kolomogorov's lemma,

$$
\begin{aligned}
I_{\text {glob }}^{1 / 2} & \lesssim \alpha \sum_{j=1}^{\infty} 2^{-j\left(2 m+\frac{n}{p}-n\right)} \sum_{i}\left|Q_{i}\right| \underset{y \in Q_{i}}{\operatorname{essinf}} M \tilde{H}(y)^{1 / 2} \\
& \lesssim \alpha \int_{\bigcup_{i} Q_{i}} M \tilde{H}(y)^{1 / 2} d y \\
& \lesssim \alpha\left|\bigcup_{i} Q_{i}\right|^{1 / 2}\left(\int_{\mathbb{R}^{n}} \tilde{H}(y) d y\right)^{1 / 2} \\
& \lesssim \alpha\left|\bigcup_{i} Q_{i}\right|^{1 / 2}\left(\int_{\mathbb{R}^{n}} \int_{0}^{\infty} H(y, t)^{2} \frac{d t d y}{t}\right)^{1 / 2} \\
& \lesssim\left(\alpha^{2-p} \int_{\mathbb{R}^{n}}|f(x)|^{p} d x\right)^{1 / 2} .
\end{aligned}
$$

Gathering the estimates we have obtained for $I_{\mathrm{loc}}$ and $I_{\text {glob }}$ we conclude as desired:

$$
I \lesssim \alpha^{-2}\left(I_{\text {loc }}+I_{\text {glob }}\right) \lesssim \alpha^{-2} \alpha^{2-p} \int_{\mathbb{R}^{n}}|f(x)|^{p} d x=\frac{C}{\alpha^{p}} \int_{\mathbb{R}^{n}}|f(x)|^{p} d x .
$$

3.4.2. $p>2$. We shall prove a more general result, namely (1.4). Let $m$ be a non-negative integer and set

$$
\mathcal{G}_{m, P}(f)(x)=\left(\iint_{|x-y|<t}\left|t \nabla_{y, t}\left(\left(t^{2} L\right)^{m} e^{-t L^{1 / 2}}\right) f(y)\right|^{2} \frac{d y d t}{t^{n+1}}\right)^{1 / 2} .
$$

We begin with a series of results that are concerned with functions of $L$ in tent spaces. Then, we shall deal with $\mathcal{G}_{m, P}$.

Consider the notation of [Aus, p. 10]. Let $\phi$ be holomorphic in $\Sigma_{\mu}, \mu \in(\omega, \pi / 2)$, with $|\phi(\zeta)| \leq C(1+|\zeta|)^{-s}$ for some $s>0, C<\infty$ and all $\zeta \in \Sigma_{\mu}$. Consider for $\alpha \in \mathbb{C}$, with $\Re \alpha>0, \varphi_{\alpha}(\zeta)=\frac{\zeta^{\alpha}}{(1+\zeta)^{\alpha}} \phi(\zeta)$. Observe that

$$
\frac{\zeta^{\alpha}}{(1+\zeta)^{\alpha}}=\left(1+\zeta^{-1}\right)^{-\alpha}
$$

and since $\zeta \in \Sigma_{\mu}$ implies $\zeta^{-1} \in \Sigma_{\mu}$ and $\arg \left(1+\zeta^{-1}\right) \in(-\mu, \mu)$, we have that

$$
\sup _{\zeta \in \Sigma_{\mu}}\left|\frac{\zeta^{\alpha}}{(1+\zeta)^{\alpha}}\right| \leq e^{\mu|\Im \alpha|}
$$

Consider the linear operator, a priori defined for $L^{2}$ functions and valued in $T_{2}^{2}$,

$$
T_{\alpha} f=\left(\varphi_{\alpha}\left(t^{2} L\right) f\right)_{t>0} .
$$

In the statements below, the constants $C$ are allowed to depend on the real part of $\alpha$ but not on its imaginary part.

Lemma 3.2. For $\Re \alpha>0, T_{\alpha}$ maps $L^{p} \cap L^{2}$ to $T_{2}^{p}$ when $2 \leq p<p_{+}(L)$ with norm controlled by $C e^{\mu|\Im \alpha|}$ for any $\mu \in(\omega, \pi / 2)$.

Proof. It is enough to consider the boundedness of $T_{\alpha}$ for the vertical norm which dominates the conical one; see Proposition 2.1. In this case, this follows from the bounded holomorphic functional calculus on $L^{p}$ for $2 \leq p<p_{+}(L)$ combined with Le Merdy's theorem [LeM, Corollary 2.3]. 
Lemma 3.3. For $\Re \alpha>\frac{n}{2 p_{+}(L)}, T_{\alpha}$ maps $L^{p}$ to $T_{2}^{p}$ when $2 \leq p \leq \infty$ with norm controlled by $C e^{\mu|\Im \alpha|}$ for any $\mu \in(\omega, \pi / 2)$.

Proof. For fixed $\alpha$ it is enough to consider the case $p=\infty$ as one can then complex interpolate from CMS between $T_{2}^{2}$ and $T_{2}^{\infty}$. We claim that for any $2<q<p_{+}(L)$, and any ball $B$,

$$
\begin{aligned}
& \left(\frac{1}{|B|} \iint_{B \times\left(0, r_{B}\right)}\left|\varphi_{\alpha}\left(t^{2} L\right) f(x)\right|^{2} \frac{d x d t}{t}\right)^{1 / 2} \\
& \leq C e^{\mu|\Im \alpha|} \sum_{j=1}^{\infty} 2^{-j(2 \Re \alpha-n / q)}\left(f_{2^{j} B}|f(x)|^{2} d x\right)^{1 / 2} .
\end{aligned}
$$

We postpone the proof of the claim until the end of this subsection. Now the right hand side is dominated by the $L^{\infty}$ norm of $f$ by using $\Re \alpha>\frac{n}{2 p_{+}(L)}$ and choosing $q<p_{+}(L)$ appropriately. Then the supremum over all $B$ of the left hand side is precisely the $T_{2}^{\infty}$ norm of $T_{\alpha} f$.

Lemma 3.4. For $0<\Re \alpha \leq \frac{n}{2 p_{+}(L)}$, $T_{\alpha}$ maps $L^{p}$ to $T_{2}^{p}$ when $2 \leq p<\frac{n p_{+}}{n-2 p_{+} \Re \alpha}$.

Proof. By a result of Harboure, Torrea, and Viviani [HTV], there is a linear map $\iota$ which for all $1<p<\infty$ is an isometry from $T_{2}^{p}$ to a closed subspace of $L_{H}^{p}$, where $H=L^{2}\left(\mathbb{R}_{+}^{n+1}, \frac{d y d t}{t^{n+1}}\right)$. Thus, the maps $\iota \circ T_{\alpha}$ form an analytic family of linear operators and they are bounded from $L^{p}$ to $L_{H}^{p}$ for $(1 / p, \alpha)$ given by the above two lemmas. Stein's complex interpolation theorem (see Gra, Theorem 1.3.7]), extended to $H$-valued functions (use the linear $\mathbb{C}$-valued maps $f \mapsto\left\langle\iota \circ T_{\alpha}(f), h\right\rangle$ for any fixed $h \in H$ ), applies since the growth is controlled in $\Im \alpha$ and gives the desired range of $p$ in terms of $\Re \alpha$.

We can use the above combined with the following lemma whose proof is postponed to Section 4

Lemma 3.5. Let $m$ be a non-negative number. For $C$ depending only on ellipticity and dimension, for any function $f \in L^{2}$ and any $x \in \mathbb{R}^{n}$,

$$
\begin{aligned}
\mathcal{G}_{m, P}(f)(x) \leq m C\left(\iint_{|x-y|<2 t}\left|\left(\left(t^{2} L\right)^{m} e^{-t^{2} L} f\right)(y)\right|^{2} \frac{d y d t}{t^{n+1}}\right)^{1 / 2} \\
+C\left(\iint_{|x-y|<2 t}\left|\nabla_{y, t}\left(\left(t^{2} L\right)^{m} e^{-t^{2} L} f\right)(y)\right|^{2} \frac{d y d t}{t^{n-1}}\right)^{1 / 2} \\
+C\left(\iint_{|x-y|<2 t} \mid\left(\left.\left(t^{2} L\right)^{m}\left(e^{-t L^{1 / 2}} f(y)-e^{-t^{2} L} f\right)(y)\right|^{2} \frac{d y d t}{t^{n+1}}\right)^{1 / 2} .\right.
\end{aligned}
$$

We now conclude for $\mathcal{G}_{P}=\mathcal{G}_{0, P}$. Start from the decomposition in the previous lemma and notice that the first term vanishes since $m=0$. The second term is bounded on $L^{p}$ for $2<p<\infty$ using $\mathcal{G}_{h}$ and rescaling $t \mapsto t^{1 / 2}$ for the part with $\nabla_{y}$ and the same argument applies for the $\partial_{t}$ part because it picks up one more power of $L$ and one still has good decay in the $L^{2}-L^{2}$ off-diagonal estimates. 
For the term with $e^{-t L^{1 / 2}}-e^{-t^{2} L}$, we apply the third lemma concerned with $T_{\alpha}$ with $\phi(\zeta)=(1+\zeta)^{1 / 2} \zeta^{-1 / 2}\left(e^{-\zeta^{1 / 2}}-e^{-\zeta}\right)$ and $\alpha=1 / 2$, which gives $2 \leq p<$ $\frac{n p_{+}(L)}{n-p_{+}(L)}=p_{+}(L)^{*}$ if $p_{+}(L)<n$ or $2<p<\infty$ if $p_{+}(L) \geq n$.

For $m$ a positive integer, then the third term of the decomposition is estimated as above with $\phi(\zeta)=(1+\zeta)^{m+1 / 2} \zeta^{-1 / 2}\left(e^{-\zeta^{1 / 2}}-e^{-\zeta}\right)$ and $\alpha=m+1 / 2$, which gives $2 \leq p<\frac{n p_{+}(L)}{n-(2 m+1) p_{+}(L)}$ if $(2 m+1) p_{+}(L)<n$ or $2<p<\infty$ if $(2 m+1) p_{+}(L) \geq n$. The first term is as good as the second one, i.e., bounded on $L^{p}$ for $2<p<\infty$.

Remark 3.6. It seems that the order $\zeta^{\alpha}$ for $\varphi_{\alpha}$ at 0 governs the $p$ range for boundedness of the conical square function. But if the decay of the off-diagonal estimate is fast enough, then this information is not necessary. For example, consider the conical square function made after $\left(t^{2} L\right)^{m} e^{-t^{2} L}$ for $m$ a positive real number. When $m$ is an integer, they are bounded on $L^{p}$ for all $2<p<\infty$ because the decay is Gaussian (polynomial of some high enough degree would suffice). But when $m$ is a non-integer, then the decay is polynomial and our method gives a limited range of $p$ for small $m$ unless $p_{+}(L)=\infty$. In other words, when $p_{+}(L)<\infty$, we obtain a range of $p$ that is a discontinuous function of $m$. We do not know whether this discontinuity is a reality or an artifact of our method. We ask therefore whether $\mathcal{G}_{m, P}$ is bounded on $L^{p}$ for $2<p<\infty$ and all real $m>0$.

Proof of (3.4). We write $f=f_{\text {loc }}+f_{\text {glob }}$, where $f_{\text {loc }}=f \chi_{4 B}$. Then, using the $L^{2}$ boundedness of square functions associated with $\varphi_{\alpha}\left(t^{2} L\right)$,

$$
\begin{gathered}
\frac{1}{|B|} \iint_{B \times\left(0, r_{B}\right)}\left|\varphi_{\alpha}\left(t^{2} L\right) f_{\mathrm{loc}}(x)\right|^{2} \frac{d x d t}{t} \leq \frac{1}{|B|} \int_{\mathbb{R}^{n}}\left(\int_{0}^{\infty}\left|\varphi_{\alpha}\left(t^{2} L\right) f_{\mathrm{loc}}(x)\right|^{2} \frac{d t}{t}\right) d x \\
\leq C \frac{1}{|B|} \int_{\mathbb{R}^{n}}\left|f_{\mathrm{loc}}(x)\right|^{2} d x=C f_{4 B}|f(x)|^{2} d x
\end{gathered}
$$

It is then enough to show that

$$
\begin{aligned}
& \left(f_{B}\left|\varphi_{\alpha}\left(t^{2} L\right) f_{\text {glob }}(x)\right|^{2} d x\right)^{1 / 2} \\
& \quad \leq C e^{\mu|\Im \alpha|} \frac{t^{2 \Re \alpha}}{r_{B}^{2 \Re \alpha}} \sum_{j=2}^{\infty} 2^{-j(2 \Re \alpha-n / q)}\left(f_{2^{j+1} B}|f(x)|^{2} d x\right)^{1 / 2} .
\end{aligned}
$$

Indeed, plugging this estimate into the integral on the Carleson region, we obtain the claim.

To this end, we set $f_{j}=f \chi_{C_{j}(B)}$ with $C_{j}(B)=2^{j+1} B \backslash 2^{j} B$ so that $f_{\text {glob }}=$ $\sum_{j \geq 2} f_{j}$ and by Minkowski's and Hölder's inequalities,

$$
\left(f_{B}\left|\varphi_{\alpha}\left(t^{2} L\right) f_{\text {glob }}(x)\right|^{2} d x\right)^{1 / 2} \leq \sum_{j \geq 2}\left(f_{B}\left|\varphi_{\alpha}\left(t^{2} L\right) f_{j}(x)\right|^{q} d x\right)^{1 / q}
$$

for any $q \geq 2$. Fix $j \geq 2$ and use the representations [Aus, (2.6)-(2.7)] to estimate $\varphi_{\alpha}\left(t^{2} L\right) f_{j}$. For the $\eta_{ \pm, t}(z)$ given by [Aus, (2.7)] we find with $\nu \in(\omega, \mu)$,

$$
\left|\eta_{ \pm, t}(z)\right| \leq \frac{C t^{2 \Re \alpha}}{|z|^{\Re \alpha+1}} e^{\nu|\Im \alpha|}
$$


Next, using (3.1) in [Aus, (2.6)] for $e^{-z L}$ with $p=2$ and $2<q<p_{+}(L), E=C_{j}(B)$ and $F=B$, we easily obtain

$$
\left(f_{B}\left|\varphi_{\alpha}\left(t^{2} L\right) f_{j}(x)\right|^{q} d x\right)^{1 / q} \leq C e^{\mu|\Im \alpha|} \frac{t^{2 \Re \alpha}}{r_{B}^{2 \Re \alpha}} 2^{-j(2 \Re \alpha-n / q)}\left(f_{2^{j+1} B}|f(x)|^{2} d x\right)^{1 / 2} .
$$

We see in this last estimate the combined roles of $\Re \alpha$ and $p_{+}(L): \Re \alpha>0$ yields integrability in $t$ while $2 \Re \alpha-n / p_{+}(L)>0$ yields the summability in space.

3.4.3. Converse inequalities. We basically follow [Aus, Theorem 6.1, Step 8]. What we have proved so far applies to any operator $L$ in our class, and in particular, to $L=-\Delta$. The explicit formula for the heat kernel implies that $p_{-}(-\Delta)=1$ and $p_{+}(-\Delta)=q_{+}(-\Delta)=\infty$. Hence, we obtain the well-known estimates

$$
\left\|G_{P,-\Delta} f\right\|_{p}+\left\|G_{h,-\Delta} f\right\|_{p}+\left\|\mathcal{G}_{P,-\Delta} f\right\|_{p}+\left\|\mathcal{G}_{h,-\Delta} f\right\|_{p} \lesssim\|f\|_{p}
$$

for all $1<p<\infty$ and $f \in L^{p}$, where we have adapted the notation to indicate the operator.

The converse $\|f\|_{p} \lesssim\left\|G_{h, L} f\right\|_{p}$ is based on the following formula for $f, g \in L^{2}$ :

$$
\begin{aligned}
\int_{\mathbb{R}^{n}} f(x) \bar{g}(x) d x & =\lim _{\varepsilon \downarrow 0} \int_{\mathbb{R}^{n}} e^{-\varepsilon L} f(x) \overline{e^{\varepsilon \Delta} g(x)} d x-\lim _{R \uparrow \infty} \int_{\mathbb{R}^{n}} e^{-R L} f(x) \overline{e^{R \Delta} g(x)} d x \\
& =-\int_{0}^{\infty} \frac{d}{d t} \int_{\mathbb{R}^{n}}\left(e^{-t L} f\right)(x) \overline{\left(e^{t \Delta} g\right)(x)} d x d t \\
& =\iint_{\mathbb{R}^{n} \times(0, \infty)}(A(x)+I)\left(\nabla e^{-t L} f\right)(x) \cdot \overline{\left(\nabla e^{t \Delta} g\right)(x)} d x d t .
\end{aligned}
$$

The last equality is obtained by integration by parts in the $x$ variable after computing the time derivative. Hence, we obtain with obvious notation

$$
\left|\int_{\mathbb{R}^{n}} f(x) \bar{g}(x) d x\right| \leq\left(\|A\|_{\infty}+1\right) \int_{\mathbb{R}^{n}} G_{h, L}(f) G_{h,-\Delta}(g),
$$

so that

and it follows that

$$
\left|\int_{\mathbb{R}^{n}} f(x) \bar{g}(x) d x\right| \lesssim\left\|G_{h, L}(f)\right\|_{p}\|g\|_{p^{\prime}}
$$

$$
\|f\|_{p} \lesssim\left\|G_{h, L}(f)\right\|_{p}
$$

For $\mathcal{G}_{h, L}$ the proof is similar. Starting from the equality above, we use the averaging trick of the Introduction and then Hölder's inequality. The details are left to the reader.

For square functions based on the Poisson semigroup, the idea is the same but one needs to integrate by parts in $t$ twice:

$$
\begin{aligned}
\int_{\mathbb{R}^{n}} f(x) \bar{g}(x) d x & =-\int_{0}^{\infty} \frac{d}{d t} \int_{\mathbb{R}^{n}}\left(e^{-t L^{1 / 2}} f\right)(x) \overline{\left(e^{-t(-\Delta)^{1 / 2}} g\right)(x)} d x d t \\
= & \int_{0}^{\infty} t \frac{d^{2}}{d t^{2}} \int_{\mathbb{R}^{n}}\left(e^{-t L^{1 / 2}} f\right)(x) \overline{\left(e^{-t(-\Delta)^{1 / 2}} g\right)(x)} d x d t \\
= & \iint_{\mathbb{R}^{n} \times(0, \infty)}(A(x)+I)\left(t \nabla_{x} e^{-t L^{1 / 2}} f\right)(x) \cdot \overline{\left(t \nabla_{x} e^{-t(-\Delta)^{1 / 2}} g\right)(x)} \frac{d x d t}{t} \\
& +2 \iint_{\mathbb{R}^{n} \times(0, \infty)}\left(t \nabla_{t} e^{-t L^{1 / 2}} f\right)(x) \cdot \overline{\left(t \nabla_{t} e^{-t(-\Delta)^{1 / 2}} g\right)(x)} \frac{d x d t}{t}
\end{aligned}
$$


The last line is obtained by distributing the second derivatives in $t$ and integrating by parts in $x$ using $\frac{d^{2}}{d t^{2}}\left(e^{-t L^{1 / 2}} f\right)(x)=L\left(e^{-t L^{1 / 2}} f\right)(x)$ and similarly with $-\Delta$. The two right hand terms are controlled by both $\left\|G_{P, L} f\right\|_{p}\left\|G_{P,-\Delta} g\right\|_{p^{\prime}}$ and $\left\|\mathcal{G}_{P, L} f\right\|_{p}\left\|\mathcal{G}_{P,-\Delta} g\right\|_{p^{\prime}}$ so that the conclusion follows as above.

\section{Proof of Lemma 3.5}

If $m=0$ we take $f \in L^{2}$ and set $f_{0}=f$. If $m \geq 1$, as the domain of $L^{m}$ is dense in $L^{2}\left(\mathbb{R}^{n}\right)$, it suffices to assume that $f$ is in that space and we set $f_{m}=L^{m} f$. Define $u_{m}=L^{m} e^{-t L^{1 / 2}} f=e^{-t L^{1 / 2}} f_{m}, v_{m}=L^{m} e^{-t^{2} L} f=e^{-t^{2} L} f_{m}$. Notice that

$$
t \nabla_{y, t}\left(t^{2 m} u_{m}\right)=2 m t^{2 m} v_{m} \vec{e}+2 m t^{2 m}\left(u_{m}-v_{m}\right) \vec{e}+t^{2 m}\left(t \nabla_{y, t} u_{m}\right)
$$

with $\vec{e}=(0, \ldots, 0,1)$. The first and second terms give rise respectively to the first and third terms on the right hand side of the desired inequality. Therefore it suffices to control the third term which gives a square function that is pointwise smaller than the integral

$$
I(x)=\iint\left|\nabla_{y, t} u_{m}(y, t)\right|^{2} \varphi^{2}\left(\frac{x-y}{t}\right) \frac{t^{4 m} d y d t}{t^{n-1}},
$$

where $\varphi$ is a smooth positive function with $\varphi=1$ on the unit ball $B(0,1)$, supported in the ball $B(0,2)$. To justify the calculations, for $0<r<R / 10<\infty$, let $\psi_{r, R}(t)=$ $\zeta(t / r)(1-\zeta(t / R))$, where $\zeta$ is a smooth function that satisfies $0 \leq \zeta \leq 1, \zeta(t)=0$ if $t \leq 1 / 2$ and $\zeta(t)=1$ if $t \geq 2$ and set

$$
I_{r, R}(x)=\iint\left|\nabla_{y, t} u_{m}(y, t)\right|^{2} \varphi^{2}\left(\frac{x-y}{t}\right) \psi_{r, R}^{2}(t) \frac{t^{4 m} d y d t}{t^{n-1}} .
$$

Let $B$ be the $(n+1) \times(n+1)$ block matrix $\left(\begin{array}{cc}A & 0 \\ 0 & 1\end{array}\right)$. By ellipticity, $I_{r, R}(x) \leq$ $C(\lambda) \Re \mathcal{I}_{r, R}(x)$ with

$$
\mathcal{I}_{r, R}(x)=\iint B(y) \nabla_{y, t} u_{m} \cdot \overline{\nabla_{y, t} u_{m}} \varphi^{2}\left(\frac{x-y}{t}\right) \psi_{r, R}^{2}(t) \frac{t^{4 m} d y d t}{t^{n-1}} .
$$

Next, we write

$$
\begin{aligned}
& \mathcal{I}_{r, R}(x)=\iint B(y) \nabla_{y, t} u_{m} \cdot \overline{\nabla_{y, t}\left(u_{m}-v_{m}\right)} \varphi^{2}\left(\frac{x-y}{t}\right) \psi_{r, R}^{2}(t) \frac{t^{4 m} d y d t}{t^{n-1}} \\
& \quad+\iint B(y) \nabla_{y, t} u_{m} \cdot \overline{\nabla_{y, t} v_{m}} \varphi^{2}\left(\frac{x-y}{t}\right) \psi_{r, R}^{2}(t) \frac{t^{4 m} d y d t}{t^{n-1}}=\mathcal{I}_{r, R}^{1}(x)+\mathcal{I}_{r, R}^{2}(x) .
\end{aligned}
$$

In the last integral, distribute the product $\varphi \psi$ on each gradient term and use Young's inequality with $\varepsilon$ to obtain a bound

$$
\|B\|_{\infty} \varepsilon I_{r, R}(x)+C \varepsilon^{-1} \iint_{|x-y|<2 t}\left|\nabla_{y, t} v_{m}\right|^{2} \frac{t^{4 m} d y d t}{t^{n-1}} .
$$

Using that

$$
t^{2 m}\left(t \nabla_{y, t} v_{m}\right)=t \nabla_{y, t}\left(t^{2 m} v_{m}\right)-2 m t^{2 m} v_{m} \vec{e}
$$


we can obtain

$$
\begin{aligned}
& \mathcal{I}_{r, R}^{2}(x) \leq\|B\|_{\infty} \varepsilon I_{r, R}(x) \\
& \quad+C \varepsilon^{-1} m \iint_{|x-y|<2 t}\left|t^{2 m} v_{m}\right|^{2} \frac{d y d t}{t^{n+1}}+C \varepsilon^{-1} \iint_{|x-y|<2 t}\left|\nabla_{y, t}\left(t^{2 m} v_{m}\right)\right|^{2} \frac{d y d t}{t^{n-1}} .
\end{aligned}
$$

Note that the first term can be hidden if $\varepsilon$ is small enough independently of $r, R, x$.

For $\mathcal{I}_{r, R}^{1}(x)$ we integrate by parts using the equation satisfied by $u_{m}$ to obtain

$$
\mathcal{I}_{r, R}^{1}(x)=-\iint B(y) \nabla_{y, t} u_{m} \cdot \nabla_{y, t}\left\{\frac{t^{4 m}}{t^{n-1}} \varphi^{2}\left(\frac{x-y}{t}\right) \psi_{r, R}^{2}(t)\right\} \overline{\left(u_{m}-v_{m}\right)} d y d t .
$$

Note that

$$
\nabla_{y, t}\left\{\frac{t^{4 m}}{t^{n-1}} \varphi^{2}\left(\frac{x-y}{t}\right) \psi_{r, R}^{2}(t)\right\}=\frac{t^{2 m}}{t^{(n-1) / 2}} \varphi\left(\frac{x-y}{t}\right) \psi_{r, R}(t) \frac{\theta(y, t) t^{2 m}}{t^{(n+1) / 2}},
$$

where $\theta: \mathbb{R}_{+}^{n+1} \rightarrow \mathbb{R}^{n+1}$ is a function with support in the cone defined by $|x-y|<2 t$ and is bounded independently of $x, r, R$. Hence, another application of Young's inequality with $\varepsilon$ yields a bound

$$
\mathcal{I}_{r, R}^{1}(x) \leq\|B\|_{\infty} \varepsilon I_{r, R}(x)+C \varepsilon^{-1} \iint_{|x-y|<2 t}\left|u_{m}-v_{m}\right|^{2} \frac{t^{4 m} d y d t}{t^{n+1}} .
$$

Again, the first term can be hidden if $\varepsilon$ is small enough independently of $r, R, x$. Gathering the obtained estimates we conclude that

$$
\begin{aligned}
I_{r, R}(x) \leq C(n, \lambda, \Lambda)\left(m \iint_{|x-y|<2 t}\left|t^{2 m} v_{m}\right|^{2} \frac{d y d t}{t^{n+1}}+\iint_{|x-y|<2 t}\left|\nabla_{y, t}\left(t^{2 m} v_{m}\right)\right|^{2} \frac{d y d t}{t^{n-1}}\right. \\
\left.+\iint_{|x-y|<2 t}\left|t^{2 m}\left(u_{m}-v_{m}\right)\right|^{2} \frac{d y d t}{t^{n+1}}\right) .
\end{aligned}
$$

Letting $r \downarrow 0$ and $R \uparrow \infty$, one obtains the desired estimate.

\section{ACKNOWLEDGMENTS}

This work was started years ago while the authors were all visiting the Universidad Autónoma in Madrid on the occasion of a special program in harmonic analysis and, after a latency period, finished this year while the authors were all visiting the Center for Mathematics and Applications of the Australian National University. We are very grateful to these institutions for their hospitality and financial support. Also we want to express our thanks to O. Dragičević and A. Volberg who showed us their work at an early stage.

\section{REFERENCES}

[Aus] P. Auscher, On necessary and sufficient conditions for $L^{p}$ estimates of Riesz transform associated elliptic operators on $\mathbb{R}^{n}$ and related estimates, Mem. Amer. Math. Soc. 186 (2007), no. 871. MR2292385 (2007k:42025)

[AHLMcT] P. Auscher, S. Hofmann, M. Lacey, A. McIntosh \& Ph. Tchamitchian, The solution of the Kato square root problem for second order elliptic operators on $\mathbb{R}^{n}$, Ann. of Math. (2) 156 (2002), no. 2, 633-654. MR1933726 (2004c:47096c)

[AM] P. Auscher \& J.M. Martell, Weighted norm inequalities, off-diagonal estimates and elliptic operators. Part I: General operator theory and weights, Adv. Math. 212 (2007), no. 1, 225-276. MR2319768(2008m:42015) 
[Be1] F. Bernicot, Use of abstract Hardy spaces, real interpolation and applications to bilinear operators, Math. Z. 265 (2010), no. 2, 365-400. MR2609316(2011c:42058)

[Be2] F. Bernicot, Use of Hardy spaces and interpolation, C. R. Math. Acad. Sci. Paris 346 (2008), no. 13-14, 745-748. MR2427074(2009h:46041)

[BZ] F. Bernicot \& J. Zhao, New abstract Hardy spaces, J. Funct. Anal. 255 (2008), no. 7, 1761-1796. MR2442082(2009k:46043)

[CMS] R. Coifman, Y. Meyer \& E.M. Stein, Some new function spaces and their applications to harmonic analysis, J. Funct. Anal. 62 (1985), no. 2, 304-335. MR791851 (86i:46029)

[CMP1] D. Cruz-Uribe, J. M. Martell \& C. Pérez, Extensions of Rubio de Francia's extrapolation theorem, Collect. Math. Vol. Extra (2006), 195-231. MR2264210 (2008a:42014)

[CMP2] D. Cruz-Uribe, J. M. Martell \& C. Pérez, Weights, extrapolation and the theory of Rubio de Francia. Operator theory: Advances and Applications, 215 Birkhäuser/Springer Basel AG, Basel, 2011. MR2797562

[DJK] B. Dahlberg, D. Jerison, \& C. Kenig, Area integral estimates for elliptic differential operators with nonsmooth coefficients, Ark. Mat. 22 (1984), no. 1, 97-108. MR735881 (85h:35021)

[DV] O. Dragičević \& A. Volberg, Bilinear embedding theorem for real elliptic differential operators in divergence form with potential, to appear in J. Funct. Anal.

[FS] C. Fefferman \& E.M. Stein, $H^{p}$ spaces of several variables, Acta Math. 129 (1972), 137-193. MR0447953(56:6263)

[Fre] J. Freshe, An irregular complex valued solution to a scalar uniformly elliptic equation, Calc. Var. Partial Differential Equations 33 (2008), no. 3, 263-266. MR2429531 (2009h:35084)

[Gar] J. García-Cuerva, An extrapolation theorem in the theory of $A_{p}$-weights, Proc. Amer. Math. Soc. 87 (1983), 422-426. MR684631 (84c:42028)

[Gra] L. Grafakos, Classical and modern Fourier analysis, Pearson Education, Inc., Upper Saddle River, NJ, 2004. MR2449250

[HTV] E. Harboure, J.-L. Torrea \& B. Viviani, A vector-valued approach to tent spaces, J. Analyse Math. 56 (1991), 125-140. MR1243101 (94i:42019)

[HM] S. Hofmann \& S. Mayboroda, Hardy and BMO spaces associated to divergence form elliptic operators, Math. Ann. 344 (2009), no. 1, 37-116. MR2481054(2009m:42038)

[HMMc] S. Hofmann, S. Mayboroda \& A. McIntosh, Second order elliptic operators with complex bounded measurable coefficients in $L^{p}$, Sobolev and Hardy spaces, Ann. Sci. Ecole Norm. Sup. série 4 44, fascicule 5 (2011).

[JK] D. Jerison \& C. Kenig, The Dirichlet problem in nonsmooth domains, Ann. of Math. (2) 113 (1981), no. 2, 367-382. MR607897 (84j:35076)

[LeM] C. Le Merdy, On square functions associated to sectorial operators, Bull. Soc. Math. France 132 (2004), no. 1, 137-156. MR2075919(2005i:47026)

[Rub] J.L. Rubio de Francia, Factorization theory and $A_{p}$ weights, Amer. J. Math. 106 (1984), 533-547. MR745140 (86a:47028a)

[St] E.M. Stein, Singular integrals and differentiability properties of functions, Princeton Mathematical Series, No. 30, Princeton University Press, Princeton, N.J., 1970. MR0290095(44:7280)

Laboratoire de Mathématiques, UMR 8628, Université Paris-Sud, Orsay F-91405; CNRS, ORsay, F-91405 France

E-mail address: pascal.auscher@math.u-psud.fr

Department of Mathematics, University of Missouri, Columbia, Missouri 65211

E-mail address: hofmann@math.missouri.edu

Instituto de Ciencias Matemáticas CSiC-UAM-UC3M-UCM, Consejo Superior de Investigaciones Científicas, C/ Nicolás Cabrera, 13-15, E-28049 Madrid, Spain

E-mail address: chema.martell@icmat.es 